\title{
THE GENERAL THEORY OF FACTORIAL SERIES*
}

\section{BY TOMLINSON FORT}

1. Introduction. The subject of this paper is factorial series, a class of series which it seems to me are more interesting than American mathematicians generally realize and which can be made to play a most important part in analysis.

By a factorial series we shall understand a series of the form

$$
c_{0}+\sum_{n=1}^{\infty} c_{n} \frac{(n-1) !}{z(z+1) \cdots(z+n-1)}=\Omega(z),
$$

where $c_{n}$ is a sequence of constants and $z$ a complex variable. The term is sometimes also made to include series in noninverted factorials or the so-called binomial-coefficient series. I shall use the term only as applying to series (1) and at times to certain generalizations of which I shall speak presently. The terms of (1) are not defined when $z=0,-1,-2, \cdots$. Such points will be understood throughout the paper as excepted in all theorems, and neighborhoods of them in all theorems relating to uniformity.

We notice the simple form of series (1), and comparison with series of negative powers, $c_{0}+\sum_{n=1}^{\infty} c_{n}(n-1) ! z^{-n}$, immediately is suggested. We observe first that the terms of the series of negative powers after the first, but for coefficient, come from successive differentiations of $1 / z$. The terms of the factorial series come from taking successive differences of $1 / z$. The power series yields itself readily to differentiation and integration and certainly a large part of its usefulness in analysis comes from this fact. The factorial series (1) is not so readily differentiated or integrated. However,

$$
\Delta \Omega(z)=\Omega(z+1)-\Omega(z)=-\sum_{n=1}^{\infty} c_{n} \frac{n !}{z(z+1) \cdots(z+n)},
$$

another factorial series of equally simple form, quite a parallel

* An address read before the Society, at the invitation of the program committee, October 26, 1929. 
to the differentiation of the power series. The application of the inverse operator, $\sum$, is also immediate. We consequently expect the factorial series to play an important role in that portion of analysis in which these operations are prominent, as for example in the theory of difference equations. We next notice that, whereas the denominators in the power series are successive integral powers of $z$, in series (1) the successive factors in the denominators, with the possible exception of a finite number at first, increase in absolute value. Hence, we expect series (1) to have certain rapidity of convergence features not possessed by the power series. This is in reality true and it is a fact that many functions asymptotic to divergent series of negative powers or at most obtainable from them by indirect summation methods are representable by convergent factorial series. It is this that gives to factorial series one of their greatest elements of interest. Factorials are asymptotic to exponentials and we expect the series to have properties in common with Dirichlet series.

The form of series (1) is simple. This is what makes it interesting. Generalizations will come to the mind of everyone. Much has been done along this line; sometimes with particular purposes in view and sometimes just to generalize. However, most of the generalizations have sacrificed not only simplicity but some useful property as well. Some generalizations that have been made are as follows. These are not necessarily fundamentally different but seem different in originating idea.

I. The series

$$
c_{0}+\sum_{n=1}^{\infty} c_{n} \frac{\omega^{n-1}(n-1) !}{z(z+\omega) \cdots(z+(n-1) \omega)}
$$

has been extensively studied by Nörlund.* He has found it useful for studying the function represented by a factorial series.

II. Replace the sequence $0,1,2, \cdots$ by a more general sequence, so that (1) becomes

$$
c_{0}+\sum_{n=1}^{\infty} c_{n} \frac{\lambda_{1} \cdots \lambda_{n}}{z\left(z+\lambda_{1}\right) \cdots\left(z+\lambda_{n}\right)} .
$$

* Acta Mathematica, vol. 37 (1914), pp. 327-87. 
This series, with various degrees of generality for the sequence $\lambda_{n}$, has been studied by a number of authors among them particularly Landau,* Pincherle $\dagger$ and myself. $\ddagger$

III. Replace each factor $(z+k)$ of the denominator by a function $S_{k}(z)$, where

and

$$
S_{1}(z)=\frac{a z+b}{c z+d}, \quad(a d-c b \neq 0),
$$

$$
S_{k}(z)=\frac{a S_{k-1}(z)+b}{c S_{k-1}(z)+d}, \quad(k>1) .
$$

This has been done by Carmichael. $\S$

IV. Replace the terms of (1) by certain general functions so that the series will include both ordinary Dirichlet series and factorial series. This was done by me a few years ago in the Transactions. $\uparrow$

V. Replace the terms of (1) by a certain general function having asymptotic properties in common with the terms of (1). This has been done by Carmichael\| in a series of papers.

VI. Replace the denominators in the terms of (1) by general polynomials in $z$ of successively ascending powers.

This was done at least by Appell** although only secondarily as a generalization of factorial series.

VII. Replace series (1) by series of a general type where the functions represented by the series can be represented by Laplace's integral. This has been done by various authors $\dagger \dagger$ over a long period, although not necessarily aiming directly at

* Münchener Berichte, vol. 36, pp. 151-218.

$\ddagger$ Palermo Rendiconti, vol. 37 , pp. 379-390.

$\dagger$ Transactions of this Society, vol. 31, pp. 233-240. For additional references see Hilb and Szasz: Encyklopädie, Band $\mathrm{II}_{3}$, Heft 8, pp. 1268-1272.

§ American Journal of Mathematics, vol. 36 (1914), pp. 267.

If Transactions of this Society, vol. 23, pp. 26-29.

II Transactions of this Society, vol. 17 (1916), p. 207.

American Journal of Mathematics, vol. 39 (1917), p. 385.

American Journal of Mathematics, vol. 40 (1918), p. 113.

American Journal of Mathematics, vol. 42 (1920), p. 76.

This Bulletin, vol. 23 (1917), p. 407.

** Bulletin de la Société Mathématique de France, vols. 48, 51.

†† For example Landau, loc. cit., Nielson, Annales de l'Ecole Normale Supérieure, vol. 19 (1902), pp. 409-453; Pincherle, Bologna Rendiconti, vol. 8 (1904), pp. 5-13; Pincherle, Accademia dei Lincei Rendiconti 1902, pp. 140-141, etc. 
factorial series. I mention in particular Widder* who delivered a paper before this Society, February, 1928, on this general topic.

I shall proceed to review briefly the present state of the theory of series (1) and (2).

2. Convergence. For the saving of time, the history of convergence theorems will not be traced in detail or completeness.

It is now classic and well known that the region of convergence of (1) is a half-plane bounded by a line perpendicular to the axis of reals. This theorem can be proved in various ways. The same theorem holds for (2), if we put the following restrictionst on $\lambda_{n}=\alpha_{n}+\beta_{n} i: \alpha_{n} \rightarrow+\infty, \beta_{n} / \alpha_{n} \rightarrow 0$. In case $\lambda_{n}$ approaches a limit, the region of convergence of (2) is bounded by a circle. $\ddagger$

Pincherle $\S$ considered (2), not requiring that $\beta_{n} / \alpha_{n} \rightarrow 0$ but only that $\left|\arg \lambda_{n}\right|<\chi<\pi / 2$ and that $\sum_{n=1}^{\infty} 1 /\left|\lambda_{n}\right|$ diverge and $\sum_{n=1}^{\infty} 1 /\left|\lambda_{n}\right|^{2}$ converge. He proves that if (2) converges when $z=z_{0}$ it converges at all points $z$ such that $\left|\arg \left(z-z_{0}\right)\right|$ $\leqq \pi / 2-\chi$. He does not argue that this exhausts the region of convergence.

However, as uniform convergence is of paramount interest when we consider series, I shall pass to theorems on uniform convergence.

Landau (loc. cit.) proved that (2) with $\lambda_{n}$ real, $\lambda_{n} \rightarrow+\infty$, converges uniformly over any finite region interior to its halfplane of convergence. Writing in the Acta Mathematica, Nörlund (loc. cit.) proved that (1) converges uniformly over any half-plane interior to its half-plane of convergence and lying to the right of the axis of imaginaries, that is, if (1) converges when $z=x_{0}>0$ it converges uniformly when $R(z) \geqq x_{0}+\delta$, $\delta>0$. In his book\| he states this same theorem without proof and does not mention the restriction that the half-plane of uniform convergence lie to the right of the axis of imaginaries. The method employed by Nörlund in the Acta Mathematica is by a transformation which seems particular in character and not

* This Bulletin, vol. 34 (1928), p. 259.

† Schnee, Berliner Dissertation, Göttingen, 1908, p. 74 ff.

$\ddagger$ Jensen, Tidsskrift for Mathematik, 1884, p. 63-72.

\& Palermo Rendiconti, vol. 37 (1914), p. 379.

|| Differenzenrechnung, p. 258. 
to apply to the more general series (2). Landau proved his theorem by a lemma which on account of its great importance in the theory of factorial series I shall now state. By means of it all known theorems on the convergence of factorial series can be proved, as well as the fundamental convergence theorems for Dirichlet series, power series, etc. This lemma was proved in various forms by Dedekind,* du Bois Reymond $\dagger$ and Cahen. $\ddagger$ To avoid repetition it is stated in general form for uniform convergence.

LEMMA 1. Hypotheses: (i) $a_{n}(z)$ and $b_{n}(z)$ are defined at all points of a set $R$; (ii) $\sum_{n=1}^{\infty} a_{n}(z)$ converges uniformly over $R$; (iii) there exists a fixed $m$ and $M$ such that $\left|b_{m}(z)\right|$ $+\sum_{n=m}^{\infty}\left|\Delta b_{n}(z)\right|<M$ for all $z$ 's of $R$. Conclusion: $\sum_{n=1}^{\infty} a_{n}(z) b_{n}(z)$ converges uniformly over $R$.

By means of Lemma 1, I have been able to establish more general theorems on uniform convergence than were previously known.

In a recent paper, $\S$ I proved the following theorem with reference to series (2).

Restrict $\lambda_{n}=\alpha_{n}+\beta_{n} i$ as follows. Require that $\alpha_{n} \rightarrow+\infty$, $\sum_{n=1}^{\infty}\left(1 / \lambda_{n}\right)$ diverge and that $\sum_{n=1}^{\infty}\left(\beta_{n} / \alpha_{n}\right)^{2}$ converge. Under these restrictions, if (2) converges when $z=z_{0}=x_{0}+y_{0} i$, it converges uniformly over any angular region defined by $x \geqq x_{0}$ and $\left|y-y_{0}\right| \leqq a\left(x-x_{0}\right)^{m}, m$ any positive integer and a any positive number.

Under these same restrictions on $\lambda_{n}$, I am now able not only to extend Nörlund's theorem of the half-plane of uniform convergence to series (2), which I was not able to do at that time, but to rid the theorem of the unnatural restriction that the halfplane of uniform convergence lie wholly to the right of the axis of imaginaries.

Let us suppose the series to converge when $z=z_{0}=x_{0}+y_{0} i$.

* Zahlentheorie, 2 d ed., p. 373.

$\dagger$ Neue Lehrsatz über die Summen unendlicher Reihen, Auftrittsprogramm, 1870, p. 10.

$\ddagger$ Annales de l'Ecole Normale Supérieure, vol. 8, p. 79.

$\S$ Transactions of this Society, vol. 31 (1929), p. 233. 
Let $z_{1}=x_{1}+y_{1} i$ be fixed and such that $x_{1}>x_{0}$, and let $z^{\prime}=x^{\prime}+y^{\prime} i$ be a variable, and require that $x^{\prime} \geqq x_{1}$. Let

and

$$
a_{n}(z)=c_{n} \frac{\lambda_{1} \cdots \lambda_{n}}{\left(z_{0}+\lambda_{1}\right) \cdots\left(z_{0}+\lambda_{n}\right)},
$$

We find that

$$
b_{n}\left(z_{0}, z\right)=\frac{\left(z_{0}+\lambda_{1}\right) \cdots\left(z_{0}+\lambda_{n}\right)}{\left(z+\lambda_{1}\right) \cdots\left(z+\lambda_{n}\right)} .
$$

$$
\frac{\Delta b_{n}\left(z_{0}, z^{\prime}\right)}{\Delta b_{n}\left(z_{0}, z_{1}\right)}=\frac{z_{0}-z^{\prime}}{z_{0}-z_{1}} \cdot \frac{z_{1}+\lambda_{1}}{z^{\prime}+\lambda_{1}}\left[\frac{\left(z_{1}+\lambda_{2}\right) \cdots\left(z_{1}+\lambda_{n+1}\right)}{\left(z^{\prime}+\lambda_{2}\right) \cdots\left(z^{\prime}+\lambda_{n+1}\right)}\right],
$$

but $\left[\left(z_{0}-z^{\prime}\right) /\left(z_{0}-z_{1}\right)\right]\left[\left(z_{1}+\lambda_{1}\right) /\left(z^{\prime}+\lambda_{1}\right)\right]$, as a function of $z^{\prime}$, remains finite over the half-plane $x^{\prime} \geqq x_{1}$. The expression in the bracket is $b_{n}\left(z_{1}, z^{\prime}\right)$ if the series $\lambda_{1}, \cdots, \lambda_{n}$ be replaced by $\lambda_{2}, \cdots, \lambda_{n+1}$. I proved in the Transactions paper just referred to that this remains uniformly finite over the region in question from which neighborhoods of those points $-\lambda_{2},-\lambda_{3}, \cdots$ which lie in it have been removed. Hence, owing to the convergence of $\sum_{n=1}^{\infty}\left|\Delta b_{n}\left(z_{0}, z_{1}\right)\right|$, as also established in my Transactions paper, we see that $\sum_{n=1}^{\infty}\left|\Delta b_{n}\left(z_{0}, z^{\prime}\right)\right|$ converges uniformly over the half-plane $x^{\prime} \geqq x_{1}$. Moreover, $b_{n}\left(z_{0}, z^{\prime}\right)$ remains finite over this half-plane. Hence, by the lemma, (2) converges uniformly over the half-plane $x \geqq x_{1}$ and Nörlund's theorem is extended to this more general series.

This theorem with the theorem quoted from my paper of last April seems to settle in a reasonably satisfactory way the question of uniform convergence for (1), and (2) with $\lambda_{n}$ restricted as indicated. It is to be noticed that the last theorem does not entirely replace the first.

The restriction that $\sum_{n=1}^{\infty}\left(1 / \lambda_{n}\right)$ diverge is a natural one, for in the contrary case it can be readily proved* that (2) either converges at all points or diverges at all points. The points $-\lambda_{1}$, $-\lambda_{2}, \cdots$ are, of course, excepted here as elsewhere in the paper.

If we do not impose the restriction that $\sum_{n=1}^{\infty}\left(\beta_{n} / \alpha_{n}\right)^{2}$ converge but in its place only require that $\beta_{n} / \alpha_{n} \rightarrow 0$, the best that I have been able to do for a region of uniform convergence is the result given in my paper of last April, namely, the familiar angular region determined by $x \geqq x_{0}$ and $\left|y-y_{0}\right| \leqq a\left(x-x_{0}\right)$.

* Schnee, loc. cit. 
3. Summability. The summability according to the CesàroHölder method of series (1), that is, the simple factorial series, was treated by Bohr.* His method of proof is by a generalization of Lemma 1. He finds that there exists an abscissa of summability analogous to the abscissa of convergence and he proves the series uniformly summable over any finite region within its corresponding half-plane of summability. A somewhat similar line of attack can be applied to the more general series (2) for summability by Riesz weighted means. I proceed to do this.

I define summation by Riesz weighted means as follows. Given $\sum_{n=0}^{\infty} a_{n}$ and $\lambda_{1}, \lambda_{2}, \cdots$. Let $\sigma_{n}=\sum_{n=1}^{n}\left(1 / \lambda_{n}\right)$ and $s_{n}=\sum_{n=0} a_{n} ;$ and let

$$
s_{n}^{(1)}=\frac{1}{\sigma_{n+1}}\left[\frac{1}{\lambda_{1}} s_{0}+\frac{1}{\lambda_{2}} s_{1}+\cdots+\frac{1}{\lambda_{n+1}} s_{n}\right] .
$$

If $s_{n}{ }^{(1)}$ approaches a limit, that limit will be called the sum of order 1 of $\sum_{n=1}^{\infty} a_{n}$ by Riesz means weighted according to the sequence $1 / \lambda_{n}$. The method clearly can be iterated to the $r$ th order. I treat first only the sum of order 1 . To do this I generalize Lemma 1.

Lemma 2. Hypotheses: (i) $a_{n}(z)$ and $b_{n}(z)$ are defined at all points of a set $R$.

(ii) The sum

$$
s_{n}^{(1)}(z)=\frac{1}{\sigma_{n+1}} \sum_{n=0}^{n} \frac{1}{\lambda_{n+1}} \sum_{n=0}^{n} a_{n}(z)
$$

approaches a limit uniformly over $R$ when $n \rightarrow \infty$.

(iii) The quantity

$$
\begin{aligned}
\left|b_{n+2}(z)\right| & +\frac{1}{\left|\sigma_{n+1}\right|} \sum_{n=0}^{n}\left|\Delta b_{n+1}(z)\right|\left|\sigma_{n+1}\right| \\
& +\frac{1}{\left|\sigma_{n+1}\right|} \sum_{n=0}^{n}\left|\frac{\lambda_{n+2}}{\lambda_{n+1} \mid}\right| \Delta b_{n+1}(z)|| \sigma_{n+1} \mid \\
& +\frac{1}{\left|\sigma_{n+1}\right|} \sum_{n=0}^{n} \frac{1}{\left|\lambda_{n+1}\right|} \sum_{n=0}^{n}\left|\Delta \lambda_{n+1} \Delta b_{n}(z)\right|\left|\sigma_{n+1}\right|
\end{aligned}
$$

remains uniformly finite over $R$.

* Göttinger Nachrichten, 1909, p. 247. 
Conclusion: $\sum_{n=0}^{\infty} a_{n}(z) b_{n}(z)$ is uniformly summable of order 1 over $R$ by this method.

This lemma is readily proved if we apply summation by parts twice to

$$
\frac{1}{\sigma_{n+1}} \sum_{n=0}^{n} \frac{1}{\lambda_{n+1}} \sum_{n=0}^{n} a_{n}(z) b_{n}(z) \text {. }
$$

It will be applied to series (2), where we again impose the restrictions on $\lambda_{n}=\alpha_{n}+\beta_{n} i, \alpha_{n} \rightarrow+\infty, \sum_{n=1}^{\infty}\left(1 / \lambda_{n}\right)$ diverges and $\sum_{n=1}^{\infty}\left(\beta_{n} / \alpha_{n}\right)^{2}$ converges. I have proved the following theorem under these hypotheses.

If (2) is summable of order 1 at $z_{0}=x_{0}+y_{0} i$, it is uniformly summable of order 1 throughout an angular region defined by $x \geqq x_{0}$ and $\left|y-y_{0}\right| \leqq m\left(x-x_{0}\right)$, where $m$ is any positive constant.

The principal steps only in the proof will be given.

We assume $x_{0}>0$ and $\alpha_{1}>0$ which entails no loss of generality. Let $b_{0}(z)=1$, and when $n>0$,

$$
b_{n}(z)=b_{n}\left(z_{0}, z\right)=\frac{\left(z_{0}+\lambda_{1}\right) \cdots\left(z_{0}+\lambda_{n}\right)}{\left(z+\lambda_{1}\right) \cdots\left(z+\lambda_{n}\right)}
$$

Let $a_{0}(z)=c_{0}$, and when $n>0$,

$$
a_{n}(z)=c_{n} \frac{\lambda_{1} \cdots \lambda_{n}}{\left(z_{0}+\lambda_{1}\right) \cdots\left(z_{0}+\lambda_{n}\right)} .
$$

Substitute in formula (3). We wish to prove that the resulting expression remains less than a constant. To show this we take the ratio of each summand in (3) to the corresponding summand with $z$ replaced by its real part $x$, and $\lambda_{n}$ replaced by its real part $\alpha_{n}$, and $\sigma_{n+1}$ by $\tau_{n+1}=\sum_{n=1}^{n+1}\left(1 / \alpha_{n}\right)$. Each of these ratios remains less than a constant. I refer to Preliminary Theorems 1 and 2 of my own paper (Transactions of this Society, vol. 31 (1929), p. 233), only remarking that

$$
\Delta \lambda_{n+1} \Delta b_{n}\left(z_{0}, z\right)=\left(z_{0}-z\right) \Delta b_{n+1}\left(z_{0}, z\right) .
$$

Moreover we have 


$$
\begin{aligned}
\left|b_{n+2}\left(x_{0}, x\right)\right| & +\frac{1}{\tau_{n+1}} \sum_{n=0}^{n}\left|\Delta b_{n+1}\left(x_{0}, x\right)\right| \tau_{n+1} \\
& +\frac{1}{\tau_{n+1}} \sum_{n=0}^{n} \frac{\alpha_{n+2}}{\alpha_{n+1}}\left|\Delta b_{n+1}\left(x_{0}, x\right)\right| \tau_{n+1} \\
& +\frac{1}{\tau_{n+1}} \sum_{n=0}^{n} \frac{1}{\alpha_{n+1}} \sum_{n=0}^{n}\left|\Delta \alpha_{n+1} \Delta b_{n}\left(x_{0}, x\right)\right| \tau_{n+1} \\
=b_{n+2}\left(x_{0}, x\right) & -\frac{1}{\tau_{n+1}} \sum_{n=0}^{n} \Delta b_{n+1}\left(x_{0}, x\right) \tau_{n+1} \\
& -\frac{1}{\tau_{n+1}} \sum_{n=0}^{n} \frac{\alpha_{n+2}}{\alpha_{n+1}} \Delta b_{n+1}\left(x_{0}, x\right) \tau_{n+1} \\
& +\frac{1}{\tau_{n+1}} \sum_{n=0}^{n} \frac{1}{\alpha_{n+1}} \Delta \alpha_{n+1} \Delta b_{n}\left(x_{0}, x\right) \tau_{n+1} \\
=\frac{1}{\tau_{n+1}} \sum_{n=0}^{n} \frac{1}{\alpha_{n+1}} \sum_{n=0}^{n} k_{n} b_{n}\left(x_{0}, x\right), & \left(k_{0}=1, \quad k_{n}=0\right),
\end{aligned}
$$

when $n>0$; that is, the $k_{n}$ 's are the coefficients of that series (2) for which $s^{(1)}\left(x_{0}\right) \equiv 1$, which is the series for which

$$
s_{n}^{(1)}(x) \equiv 1 ; \frac{1}{\tau_{n+1}} \sum_{n=0}^{n} \frac{1}{\alpha_{n+1}} \sum_{n=0}^{n} k_{n} b_{n}\left(x_{0}, x\right)=1 ;
$$

and our theorem follows by Lemma 2 .

There is a half-plane of summability in every way analogous to the half-plane of convergence. This theorem follows from the preceding as in the corresponding case for convergence.

The generalization of Lemma 2 to a weighted sum of any fixed number of iterations is straightforward. The formulas, however, are increasingly complicated. When we come to apply these lemmas to factorial series, I find a difficulty in proving that the ratio of each summand in $z$ and $\lambda_{n}$ to the corresponding summand in $x$ and $\alpha_{n}$ remains uniformly finite. I find that I must assume in addition to the assumption already made that $\lambda_{n+1} / \lambda_{n}$ remains finite and that $\Delta\left(\lambda_{n+1} / \lambda_{n}\right) \rightarrow 0$ and that $x-x_{0}>\delta>0$. I am then able to establish theorems for $r$ th order summation by weighted means analogous to those just proved for first order summation by weighted means, namely uniform summation over an angular region bounded by straight lines 
from which the half-plane of summation follows. The additional assumptions on $\lambda_{n}$ and the assumption $\left(x-x_{0}\right)>\delta$ are probably incident to the method of proof. Lines of summability follow immediately.

It is to be noticed, however, that all these results are more general than those of Bohr (loc. cit.) even for the simple series (1) which he treats and the Cesàro-Hölder method which he uses. He only proves uniform summability over a finite region and he retains the restriction $x-x_{0}>\delta$.

4. Equiconvergence Theorems. Landau (loc. cit.) proved in his classical memoir that series (1) and the ordinary Dirichlet series

$$
\sum_{n=1}^{\infty} c_{n} n^{-2}
$$

where the coefficients are the same, converge at the same points. Kluyver* had already proved a similar theorem for absolute convergence.

In the Transactions of this Society for January, 1922, I set up the series

$$
\sum_{n=1}^{\infty} c_{n} \frac{k}{z+k-1} \frac{\Gamma(n k)}{\Gamma(z+n k)} \cdot \frac{\Gamma(z+k)}{\Gamma(k)} .
$$

This series reduces to the ordinary factorial series for $k=1$ and has the ordinary Dirichlet series (4) as a limiting series when $k \rightarrow \infty$. It is found that by placing only slight restriction on $k$ we have a wide class of series including the limiting case (Dirichlet series) for which the equiconvergence theorem holds. That is, (5) converges at the same points for the values of $k$ including the limiting case where $k \rightarrow \infty$.

Pincherle (loc. cit.) proved (2) and

$$
\sum_{n=1}^{\infty} c_{n} e^{-\sigma_{n} z}, \quad \sigma_{n}=\sum_{n=1}^{n} \frac{1}{\lambda_{n}}
$$

equiconvergent provided, among other restrictions, $\sum_{n=1}^{\infty}\left(1 /\left|\lambda_{n}\right|\right)^{2}$, converges. In my paper in the Transactions of April, I removed all restrictions on $\lambda_{n}$ except that $\sum_{n=1}^{\infty}\left(1 / \lambda_{n}\right)^{s+1}$ be convergent and proved an equiconvergence theorem for (2) and for the series $\sum_{n=1}^{\infty} c_{n} e^{-\sigma_{1 n}}$, where

* Nieuw Archiv, (2), vol. 4 (1899), p. 74. 
$g_{1 n}=\left(\sum_{n=1}^{n} \frac{1}{\lambda_{n}}\right) z-\left(\sum_{n=1}^{n} \frac{1}{\lambda_{n}^{2}}\right) z^{2}+\cdots+(-1)^{s-1}\left(\sum_{n=1}^{n} \frac{1}{\lambda_{n}^{s}}\right) z^{8}$.

In another theorem in the same paper I proved that, if $\lambda_{n}$ is real, $\lambda_{n} \rightarrow+\infty$ and $\sum_{n=1}^{\infty}\left(1 / \lambda_{n}\right)$ diverges, then (2) and the Dirichlet series, $\sum_{n=1}^{\infty} c_{n} e-\sigma_{n} z, \sigma_{n}=\sum_{n=1}^{n}\left(1 / \lambda_{n}\right)$, have the same line of convergence. The location of the lines of convergence is now given by the familiar formulas for Dirichlet series.

Equisummability theorems analogous to the above for summation by weighted means hold at least if we put the restrictions of $\S 4$ on $\lambda_{n}$. Bohr (loc. cit.) has results for the simple factorial series (1) and the ordinary Dirichlet series using the Cesàro-Hölder summation method.

5. Function Represented. The question of the nature of the function representable by a class of series and how to obtain this representation is one of the most interesting questions in the study of series.

I have already remarked that if $\Omega(z)$ is the function represented by (1), then

$$
\Delta \Omega(z)=-\sum_{n=1}^{\infty} c_{n} \frac{n !}{z(z+1) \cdots(z+n)} .
$$

In order for the series to be of much use in the study of difference equations it is necessary to prove other theorems relative to algebraic operations with factorial series and to uniqueness of representation in factorial series. These necessary theorems have been handled principally by Nörlund (loc. cit.). The uniqueness theorem, so important in work of this kind, does not hold for the binomial coefficient series, as was shown by Pincherle, ${ }^{*}$ and this fact robs that series of much interest which attaches to factorial series. It would be interesting to investigate the situation as regards these useful theorems for series (2). This does not seem to have been done.

That $\Omega(z)$ is analytic over a region of uniform convergence is immediate. This does not include the point $\infty$, of course, which is not within the region of uniform convergence. There are poles at each of the points $-\lambda_{1},-\lambda_{2}, \cdots$, which lie within the region of convergence. The line of convergence differs from

* Atti, Accademia dei Lincei, Rendiconti, (5), vol. 2, pp. 417-426. 
the circle of convergence of a power series in that no singular point need necessarily lie upon it. As a matter of fact Nörlund (loc. cit., p. 374) has shown that if we consider series $\left(1^{\prime}\right)$ there is a line, $x=l$, which is approached by $a$, the abscissa of convergence, when $\omega \rightarrow 0$. We write $a(\omega)$. This line is characteristic of the function which ceases to be analytic at all points, or at least does not remain finite, within the band $l-\epsilon \leqq x \leqq l+\epsilon$ no matter how small $\epsilon>0$ may be.

Nörlund has shown (loc. cit., p. 347) that the behavior of $\Omega$ as $z \rightarrow \infty$ remaining in the half-plane of convergence is given by the following formulas:

$$
\begin{aligned}
& \lim \Omega(z)=c_{0}, \\
& \lim z \Omega(z)=c_{1}, \\
& \lim (z+1)\left[z \Omega(z)-c_{1}\right]=c_{2}, \\
& \lim (z+2)\left[(z+1)\left\{z \Omega(z)-c_{1}\right\}\right]=c_{3},
\end{aligned}
$$

These relations seem to give a proper description of the asymptotic behavior of the function.

Watson* has written a long paper in which his thesis is to prove that functions asymptotic to divergent power series in the sense of Poincaré,

$$
f(x) \sim a_{0}+\frac{a_{1}}{x}+\frac{a_{2}}{x^{2}}+\cdots,
$$

can be represented by a convergent factorial series. He succeeds in establishing some very general theorems. These theorems are a little long in statement and are not given in detail.

Nörlund (loc. cit., p. 379) proves that functions represented by divergent power series of the form

$$
a_{0}+\frac{a_{1}}{z}+\frac{a_{2}}{z^{2}}+\cdots,
$$

summed by Borel's integral definition can be represented by convergent factorial series.

Necessary and sufficient conditions that a function be repre-

* Palermo Rendiconti, vol. 34 (1912), p. 41. 
sentable by convergent factorial series have* been obtained. They center around Laplace's integral

$$
\int_{0}^{1} Q(t) t^{z-1} d t .
$$

6. Difference Equations. With the background of these theorems the establishment of a broad theory of analytic solutions of linear difference equations is no very difficult matter. This has been done by Nörlund in a most excellent way. The essential preliminary, however, was the satisfactory theory of factorial series. Nörlund's principal theorem on linear difference equations, in a word, is that linear difference equations whose coefficients can be developed into factorial series possess a fundamental system of analytic solutions which can be expressed in terms of factorial series. For exact statement of the theorem I refer to his book. $\dagger$ The results may also be found in his papers. This is, so far as I know, really the most important application of factorial series.

Solutions of linear differential equations under certain quite general conditions can be written as factorial series, $\ddagger$ which form has been useful in studying them in the neighborhood of singular points. This has not seemed to me, however, as interesting or natural as the difference equation results.

7. Work of Carmichael. I wish now to spend a short while discussing in greater detail the work of R. D. Carmichael (loc. cit., (2)). The researches of which I wish to speak are embodied in a series of papers beginning with the Transactions of this Society for 1916 and including three papers in the American Journal of Mathematics, and one in the Bulletin of the American Mathematical Society. This work is in the nature of a generalization but is peculiarly complete. The papers seem to have come from the asymptotic notions of Poincaré particularly as used by Birkhoff, Carmichael, and others in the study of difference equations. Lemma 1 of this paper is abundantly used. The author acknowledges his indebtedness to Landau.

* See Encyklopädie, vol. II-C-1-12, p. 1272; also Nörlund, loc. cit., p. 371.

$\dagger$ Differenzenrechnung, Berlin, 1924, p. 365.

$\ddagger$ Horn, Mathematische Annalen, vol. 71, p. 510; H. T. Davis tells me that he has found factorial series indispensible in his studies of differential equations of infinite order. 
In Landau's paper the part of $b_{n}$ of our Lemma 1 is played by

$$
\frac{z_{0}\left(z_{0}+1\right) \cdots\left(z_{0}+n-1\right)}{z(z+1) \cdots(z+n-1)}
$$

which he shows to be asymptotic to $n^{-\left(z-z_{0}\right)}$. Carmichael sets up series for which

$$
b_{n} \sim n^{\mu\left(z-z_{0}\right)}\left(t_{0}+\frac{t_{1}}{n}+\cdots\right) .
$$

It is to be noticed that this is a more general asymptotic form for $b_{n}$. This form is not featured by the author but seems to me the central formula of the papers and may have been the point of beginning of the research. If so, the problem would immediately be to generalize

$$
\frac{(n-1) !}{z(z+1) \cdots(z+n-1)}
$$

to forms which yield this asymptotic representation for the function $b_{n}$. Carmichael assumes a function

$$
g(z) \sim z^{P(z)} e^{Q(z)}\left(1+\frac{a_{1}}{z}+\frac{a_{2}}{z^{2}}+\cdots\right)
$$

analytic at least when $z$ is in a sector including the axis of reals and sufficiently large in absolute value. $P(z)$ and $Q(z)$ are polynomials. The establishment of asymptotic form (6) is now straightforward. At some points more stringent restrictions are put on $g(z)$. The existence of such functions is readily established. Carmichael's papers concern themselves with series of the type $\sum_{n=0}^{\infty} c_{n} g(z+n)$ and closely allied forms. The theory runs much as would be expected and much like the theory of factorial series. There is a half-plane of convergence, of absolute convergence, etc. with bounding lines given by expected familiar formulas. He does not treat summability of the series when divergent. However, Professor Carmichael tells me that this has been treated according to the CesàroHölder method by C. F. Green in a dissertation at the University of Illinois which has not been published. Carmichael makes considerable progress on the representation of functions by such series. He proves theorems showing that functions given 
by asymptotic power series or such series at most summable by Borel's integral method can be represented by convergent series of his functions. For exact statements of results I refer to Carmichael's papers. The asymptotic form of the function represented by such series is investigated. Again it is what we expect from known facts relative to factorial series. Of particular significance it seems to me is the author's definition of an asymptotic series using the series of his papers. It is a considerable generalization of the notion of Poincaré. More recently Ritt* has defined and studied asymptotic Dirichlet series.

Carmichael is also led to study series of the type

$$
\sum_{n=1}^{\infty} c_{n} e^{-\lambda_{n}(1) z-\lambda_{n}(2) z^{2}-\cdots-\lambda_{n}(k) z^{k}}
$$

the first time, so far as I know, that such series have been studied. He greatly restricts his parameters but even then obtains interesting results. The series is introduced only for its immediate use and the author states that he has developed a more extensive theory than he publishes.

8. Conclusion. In conclusion, numerous mathematicians have written on factorial and allied series. I regard the work of Nörlund as most significant. Next that of Landau followed by that of Pincherle and Carmichael. One should also surely mention Jensen, Bendixon, Nielson and Schnee. There have been other writers. No effort has been made to be exhaustive. My apologies are offered to those authors whose fine papers are not mentioned. Landau did not start the study of factorial series nor did he originate many of the theorems. He, however, builds a consistent theory, proving all theorems with the utmost rigor and writing with his peculiarly clear and elegant style. Nörlund takes up Landau's task and develops a more or less complete theory for the simple factorial series and what he proves to be its equivalent in many ways, namely series $\left(1^{\prime}\right)$. His interest is, probably, primarily in difference equations and his theory of factorial series lends itself well to that study. Pincherle adds a number of theorems. He is primarily concerned with series (2). I have just spoken of Carmichael's work.

Lehigh University

* American Journal of Mathematics, vol. 50 (1928), pp. 73-85. 